\title{
Evaluación del impacto social, económico y ambiental de la explo- tación de una empresa minera en las aldeas de San Andrés, San Miguel y Azacualpa, La Unión, Copán, Honduras, marzo de 2015 a febrero de 2016
}

José Salomón Orellana Peña ${ }^{1}$

\section{RESUMEN}

En el marco de las líneas prioritarias de investigación definidas por la UNAH y patrocinado por la Dirección de Investigación Científica y Posgrados (DICYP), se realizó un estudio de campo durante el periodo comprendido de marzo de 2015 a febrero de 2016, en el que se describe e interpreta la percepción que se tiene sobre el desarrollo sostenible con respecto a la industria de la minería metálica, específicamente en sus tres dimensiones discutidas en la Cumbre de Río de 1992: económica, social y ambiental.

Este trabajo plantea la revisión de las teorías que han promovido la industria de la minería y la necesidad de incorporar una cuarta dimensión del desarrollo orientada al fortalecimiento de la institucionalidad, la gobernanza y transparencia; lo que permitirá articular y armonizar las restantes dimensiones del desarrollo.

La presente investigación contempló una serie de entrevistas con diferentes actores relacionados a la industria de la minería y el desarrollo, a nivel público, privado y sociedad civil. Algunas herramientas utilizadas en este estudio cuantitativo fueron cuestionarios, entrevistas, observaciones y apuntes del investigador.

En términos generales, se estima que son más los costos sociales y ambientales que genera la industria de la minería que el beneficio económico. Los resultados demuestran también que la industria de la minería metálica no es compatible con el concepto universal del desarrollo, el cual sugiere satisfacer las necesidades del presente sin afectar las necesidades de las futuras generaciones, ya que la minería es un recurso no renovable.

\footnotetext{
${ }^{1}$ Beneficiario de una beca básica, profesor investigador, Carrera de Comercio Internacional, Centro Regional Universitario de Occidente, UNAH: Jose.orellana@unah.edu.hn
} 
Palabras claves: desarrollo sostenible, social, económico, ambiental, comunidad, minería, percepción, extractivismo, modernización, dependencia.

\section{ABSTRACT}

As part of the research priorities identified by the UNAH and sponsored by the Department of Scientific Research and graduate DICYP, a field study was conducted during March 2015 to February 2016, which describes and interprets the perception people have about sustainable development regarding industry specifically metal mining in three dimensions discussed at the 1992 Rio Summit; such as economic, social and environmental. This paper presents a review of the theories that have promoted the mining industry and the need to incorporate a fourth dimension of development aimed at strengthening the institutional, governance and transparency; in order to articulate and harmonize the other dimensions of development. This work covered a series of interviews with different actors involved in the mining industry and development, both public, private and civil society level. Some quantitative tools used in this study were questionnaires, interviews, observations, and researcher's notes. Overall it is estimated that there are more social and environmental costs generated by the mining industry than profit. The results also show that the metal mining industry is not compatible with the universal concept of development that suggests meet the needs of the present without compromising the needs of future generations to meet the need; because mining is a nonrenewable resource.

Keywords: sustainable, social, economic, environmental, community development, mining, perception, extractivism, modernization, dependency. 


\section{INTRODUCCIÓN}

Este artículo presenta una evaluación del impacto social, económico y ambiental de la explotación de una empresa minera en las aldeas de San Andrés, San Miguel y Azacualpa del municipio de La Unión, Copán, Honduras.

Se hace un análisis partiendo de los protocolos internacionales del Proyecto Minería Minerales y Desarrollo Sostenible (MMDS), organización que aglutina diferentes instituciones internacionales que se dedican a la investigación y al estudio de la industria de la minería a nivel global. El MMDS define a la minería como "un sector extenso y heterogéneo a lo largo de las etapas de exploración, producción, uso, reutilización, reciclaje y disposición final de los minerales y metales" (Sandbrook, R. 2002, p.14). En ese sentido, esta investigación aborda la temática analizando su impacto en las tres dimensiones del desarrollo sostenible: social, económico y ambiental; dado que los productos minerales son esenciales para las sociedades y economías contemporáneas. En consecuencia, muchas necesidades básicas no pueden satisfacerse sin recurrir a ellos, aunque la industria solamente responde a la demanda de productos minerales, está muy lejos de satisfacer lo que la sociedad espera de ella.

El proceso de producción y utilización de los minerales podría ayudar a la sociedad a alcanzar otros objetivos, tales como generar puestos de trabajos directos e indirectos y ayudar al desarrollo de la economía municipal y nacional. Cuando esta industria no satisface estos objetivos, se considera que no cumple con su responsabilidad social y se genera un creciente rechazo hacia ella.

\section{MÉTODO}

\section{Diseño: enfoque cuantitativo de la investigación}

Esta investigación se realizó mediante un abordaje desde la óptica cuantitativa con un diseño descriptivo, el que pretendió establecer las características, rasgos y propiedades de los eventos, sucesos o fenómenos que se estudiaron. Los estudios descriptivos fueron orientados a responder, mediante interrogantes relacionadas a las variables de estudio, las cuales ocasionaron una comprensión estadígrafa del tema. 


\section{Población y entorno}

La investigación tomó en cuenta como unidades de análisis los pobladores del municipio de San Andrés, Azacualpa, San Miguel (pobladores del municipio de La Unión, Copán, Honduras) y otra muestra final con actores vinculados al desarrollo humano sostenible del país que convergen en el sector. El interés se centró en ver qué tanto ha penetrado el discurso del desarrollo en estas comunidades, cómo lo percibe la gente, con qué asocian la palabra desarrollo sostenible y la minería, cuál es la imagen que la población tiene de este concepto.

La recopilación de información con algunos actores claves fue fundamental para plasmar la información propuesta. La entrevista con líderes de las comunidades permitió encontrar a otros actores relevantes para este estudio. Se trató de tener una igualdad de género en las aplicaciones de los instrumentos de recolección. Con los datos oficiales de la población de estas comunidades se buscó una participación representativa de la comunidad. Los cuestionarios utilizados fueron aplicados en la mayor parte de los barrios y colonias de los lugares seleccionados. La búsqueda de actores claves y líderes fue selectiva en el sector de La Unión, Copán.

\section{Intervenciones y análisis de la información}

Para esta investigación se diseñó un cuestionario de 52 preguntas, las cuales fueron abordadas para cumplir con las mediciones de dimensiones propuestas. La información de estos cuestionarios fue incluida posteriormente en una plantilla diseñada en SPSS según su codificación, definición de variables y categorías. Este programa sirvió para ordenar la información y poder extraer las dimensiones, es decir, los conceptos que la gente le fue atribuyendo a la noción del desarrollo.

Para la interpretación de los datos arrojados por el instrumento en las diferentes unidades de análisis en este contexto, se generó una matriz de datos o base de datos enumerando las dimensiones, variables y subvariables, asignándoles una leyenda para identificar a las mismas. A su vez, se procedió a determinar la escala de medición de las variables. Una vez ingresado los datos, se aplicó un tratamiento estadístico con el programa SPSS V 10 (statistical package for the social sciences). Con esta base de datos se hicieron los diferentes cálculos estadísticos y pruebas pertinentes para medir las variables de la investigación y la respectiva comprobación de la hipótesis del estudio. Las respectivas pruebas estadísticas que se realizan para contestar cada objetivo de investigación son: análisis descriptivo de las variables (frecuencias relativas y representación gráfica de las variables), interpretación descriptiva de las 
variables con base a las mediciones encontradas, relacionando los resultados de las dimensiones de estudio.

\section{FUNDAMENTACIÓN TEÓRICA}

\section{Marco contextual de la investigación: la región occidental de Honduras}

En Honduras, la actividad minera comenzó en tiempos de la colonia, hace más de 400 años, pero es durante el Gobierno de la Reforma Liberal de Marco Aurelio Soto cuando se impulsó con mayor intensidad (Pino, 2013). En la actualidad, todas las empresas mineras metálicas pertenecen a extranjeros. Asimismo, hay una serie de empresas extranjeras que están procurando obtener nuevas concesiones.

La Asociación Nacional de Mineros de Honduras (ANAMINH) y la Asociación Comercial de la Industria, tienen una lista de 23 empresas miembros, algunas de las cuales son proveedoras de servicios. Sus empresas matrices están en Canadá, Estados Unidos, Italia y China. A la ANAMIMH le interesa presentar las actividades de sus miembros de forma tan positiva como sea posible. Por ejemplo, ha colaborado con UNICEF para publicar un libro dirigido a niños hondureños encomiando los beneficios y el entendimiento de los potenciales riesgos medioambientales de la minería. Igualmente, el 22 de mayo de 2013, el Consejo de la Iniciativa de Transparencia de la Industria Extractiva (EITI) admitió a Honduras como país candidato. De conformidad con las normas de la EITI, Honduras estaba obligada a publicar su primer estándar EITI en un año y seis meses después de convertirse en un candidato es decir el 22 de noviembre de 2014 y tenía que presentar un informe de validación final a la Junta dentro de los dos años y seis meses siguientes de haberse convertido en un candidato oficial, es decir para el 22 de noviembre 2015. Hoy en día Honduras es un miembro pleno de la EITI y está comprometida en observar todas las normas relacionadas a la transparencia de la industria de la minería.

La Mina de San Andrés, la cual fue objeto de estudio en esta investigación, está ubicada en el municipio de La Unión, Copán, en el occidente de Honduras y fue operada por los colonos durante muchos años en forma artesanal, es decir, sin tecnología moderna. Sin embargo, en 1998, inició operaciones la Compañía Minera Greenstone Minerals, de capital canadiense, utilizando la técnica de explotación a cielo abierto. Esta compañía tuvo que reubicar a tres aldeas pertenecientes al municipio de La Unión, Copán; Así, San Andrés Minas, fue reubicado totalmente, San 
Miguel y Azacualpa, fueron reubicados parcialmente, aunque existe la intención de ser reubicadas totalmente.

Es en este contexto en donde inicia todo un proceso de conflictos sociales entre las poblaciones de las comunidades, empresa minera, Gobierno municipal e instituciones del Gobierno central, incluyendo muchos conflictos ambientales ocurridos en los últimos años. Desde 1998 a la fecha, la Compañía Minera San Andrés ha cambiado de concesionarios por lo menos en cinco ocasiones. Actualmente, está siendo operada por la empresa Aura Minerals y de acuerdo a Oxfam América, esta mina "produce alrededor de 70,000 onzas de oro al año" (Power, 2009 p. 28). En términos de rentabilidad financiera, la onza de oro se cotizaba en el mercado internacional en diciembre 2013 a $\$ 1,764.00$ (Santacruz, J. 2013). Esto generó una utilidad anual arriba de los 123 millones de dólares, contrastando con los Índices de Desarrollo Humano (IDH), que en el 2012 fue de 0.632 a nivel nacional, y a nivel del municipio de La Unión de Copán de 0.571 de acuerdo al Informe sobre Desarrollo Humano del Programa de las Naciones Unidas para el Desarrollo. (PNUD, 2013).

\section{MARCO CONCEPTUAL}

\section{Nociones y teorías del desarrollo}

Desarrollo sostenible: después de la Segunda Guerra Mundial, el concepto de desarrollo tuvo una interpretación meramente económica enfocado en los Estados-naciones como sujetos y dividiéndose en países desarrollados y subdesarrollados (O’Neill, 2009). Asimismo, el concepto de desarrollo sostenible surgió en gran medida por las grandes brechas y los desequilibrios del mismo concepto de desarrollo en la mayoría de los países del mundo. El flagelo de la pobreza es hoy uno de los mayores desafíos que enfrentan los países.

El Banco Mundial (2015), afirma que casi la mitad de la población mundial, unos 2,800 millones de personas, subsiste con menos de USD 2 diarios (Leary, 2015). Desde la perspectiva de la población, el desarrollo sostenible forma parte de un conjunto de ideas sobre la forma cómo los seres humanos deberían interactuar mejor entre sí y con el medioambiente. Implica integrar y cumplir con objetivos económicos, sociales y ambientales, pilares discutidos en la Cumbre de Río de 1992.

La definición más aceptada y discutida sobre el desarrollo sostenible se utilizó en 
1987 por la Comisión Mundial sobre Medio Ambiente y Desarrollo, más conocida como Comisión Brundtland, quien la definió así: "Desarrollo sostenible es el desarrollo que satisface las necesidades del presente, sin comprometer la capacidad de las futuras generaciones para satisfacer sus propias necesidades" (Armiño, 2000, p. 114). La definición original de la Comisión Brundtland puede ser interpretada en cuatro condiciones para alcanzar el desarrollo sostenible: deben satisfacerse las necesidades materiales y otras que permitan a la generación actual tener una mejor calidad de vida, con la mayor equidad posible; respetando los límites de los ecosistemas, construyendo las bases para que las futuras generaciones puedan satisfacer sus propias necesidades. Sin embargo, aplicar este concepto al sector de la industria de la minería genera ciertas contradicciones, ya que los metales son recursos no renovables y se contraponen a los principios de la sostenibilidad, especialmente en la dimensión ambiental.

\section{El desarrollo sostenible y el sector de los minerales}

El desafío del marco de desarrollo sostenible consiste en observar que el sector de los minerales en su conjunto contribuya hoy a la prosperidad y bienestar humano, sin reducir las posibilidades de futuras generaciones para hacer lo mismo. Por ende, el enfoque debe ser amplio, debe tener en cuenta todo el sistema de los minerales y con visión de futuro, lo que implica la definición de objetivos a corto y largo plazo.

Si el sector de los minerales está dispuesto a contribuir de manera positiva al desarrollo sostenible, debe demostrar un avance sostenido en su contribución social, económica y ambiental, con sistemas de gobernanza renovados y en continua evolución. El sector necesita un marco de referencia dentro del cual debería juzgar y perseguir todo tipo de desarrollo, es decir una solida institucionalidad.

En la figura siguiente se aprecia un conjunto de principios rectores para las cuatro dimensiones del desarrollo humano sostenible propuestos en esta investigación para comprender mejor el sector de la minería y el desarrollo humano sostenible. Las primeras tres dimensiones económico, social y ambiental, son los tres pilares que fueron discutidos en la Cumbre de Rio de 1992. 
Figura 1. Integralidad de las dimensiones del Desarrollo Humano Sostenible y la Institucionalidad

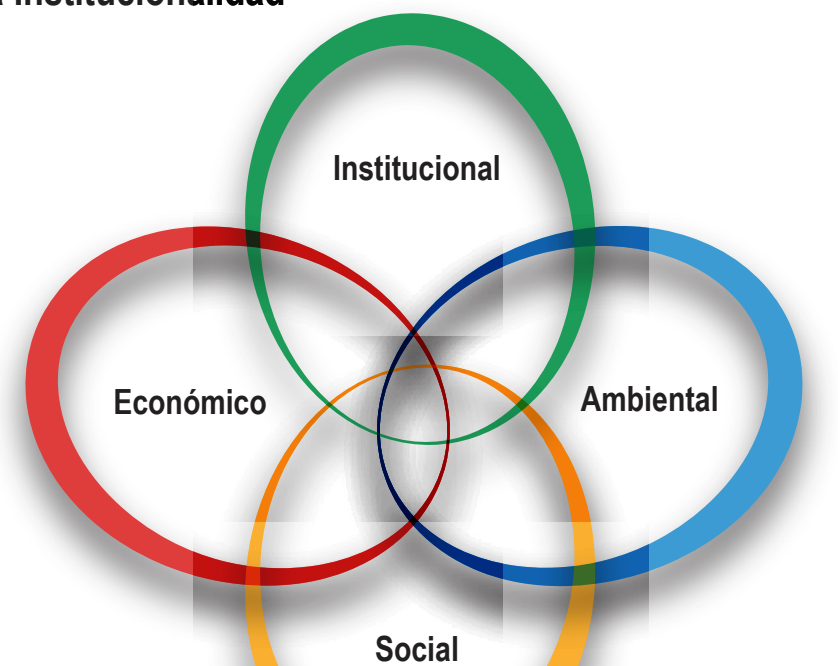

Fuente: Adaptación propia

Estos principios deben verse como aspiraciones de alto nivel, que igualmente pueden aplicarse a otros ámbitos de la economía. Deben interpretarse de manera tal que se reconozca la diversidad, los límites de los niveles actuales de conocimiento y capacitación y la sostenida necesidad de minerales que tiene la sociedad.

Los enfoques políticos, por su parte, se centran en cómo los pueblos establecen instituciones para organizar sus sociedades y de qué tipo de instituciones se trata. Los factores políticos y sociales, incluida entre estos la cultura, no pueden dejarse de lado en el estudio del desarrollo económico y, hasta cierto punto, lo condicionan de manera decisiva (Serrano, 2009). Es cierto que los economistas todavía no terminan de dominar los conceptos de crecimiento y desarrollo económico y, en consecuencia, sus recomendaciones de política pueden considerarse extremadamente moderadas $y$, desde luego, insuficientes para la resolución de un problema de tal magnitud. Pero, la economía neoclásica, basada en el funcionamiento de los mercados, sí estipula una serie de recomendaciones claras en materia de política económica y estrategias de desarrollo. El problema es que los modelos económicos suponen la existencia de un marco político y social homogéneo, neutral, estable y, en gran medida, inspirado en las modernas sociedades industriales 0 , incluso, posindustriales y posmodernas (DePaul, 2015). 
Sin embargo, la dimensión política y social del desarrollo es, en muchas ocasiones, un elemento clave en la explicación de los procesos de desarrollo o, en su caso, de no desarrollo. La economía neoclásica, basada en el funcionamiento de los mercados, estipula una serie de recomendaciones claras en materia de política económica y estrategias de desarrollo, pero que supone la existencia de un marco político y social homogéneo, neutral, estable, inspirado en el de las modernas sociedades industriales.

Alternativamente, sociólogos y antropólogos destacan las carencias de las sociedades tradicionales para obtener resultados positivos en materia de desarrollo económico: los lastres que suponen la existencia de comunidades cerradas y sus redes clientelares, el excesivo influjo de la religión, el estatus de la mujer o el de los ancianos serían todos ellos, entre muchos otros, factores que dificultan el desarrollo económico (Salinas, 1993). Por ello, es imprescindible abordar el proceso del desarrollo desde las perspectivas política y social y conocer los instrumentos conceptuales que ambas disciplinas ofrecen para su comprensión (Bebbington, 2012).

Las dos escuelas principales que han tratado la problemática del desarrollo desde la perspectiva política y social son la teoría de la modernización y la teoría de la dependencia; aunque en los últimos años aparece la denominada corriente del posdesarrollo. A finales de los años 60, apareció la teoría de la dependencia, la que rápidamente se extendió al análisis económico (Aráoz, 2009). Ambas escuelas tienen un componente marxista muy importante y tienden más bien a relacionar el subdesarrollo con las condiciones imperantes en la escena política internacional; sus conclusiones consisten en un rechazo a las virtudes de la globalización con base a consideraciones políticas y económicas. A partir de 1990 el modelo de desarrollo predominante en la industria de la minería, especialmente en América Latina es el neo-extractivismo.

El extractivismo era una modalidad de acumulación que comen?zó con la conquista y la colonización y que ahora se ha acentuado con la economía mundial: el sistema capitalista. Esta modalidad de acu $\urcorner$ mulación extractivista estuvo determinada desde entonces por las demandas de los centros metropolitanos del capitalismo naciente. Unas regiones fueron especializadas en la extracción y producción de materias primas, es decir, de bienes primarios, mientras que otras asumieron el papel de productoras de manufacturas.

El extractivismo no se limita a los minerales o al petróleo. Hay también extractivismo agrario, forestal e inclusive pesquero (Gudynas, 2009). En la actualidad la cuestión 
de los recursos naturales reno?vables debe ser enfocada a la luz de las recientes evoluciones y tendencias. Dado el enorme nivel de extracción, muchos recursos renovables, por ejemplo el forestal o la fertilidad del sue $\urcorner$ lo, pasan a ser no renovables, ya que el recurso se pierde porque la tasa de extracción es mucho más alta que la tasa ecológica de renovación del recurso. Entonces, a los ritmos actuales de extra-

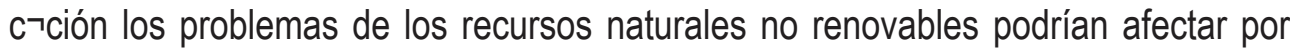
igual a todos los recursos, renovables o no. En la práctica, el extractivismo ha sido un mecanismo de sa?queo y apropiación colonial y neocolonial.

\section{MARCO FILOSÓFICO DE LA INVESTIGACIÓN}

\section{Desarrollo sostenible: presencias y ausencias en la idea del desarrollo sosteni- ble en la minería}

La industria de los minerales debe desempeñar un papel clave en el sector, colaborando con este para que haga un considerable aporte positivo al desarrollo sostenible. En el futuro podrían ocurrir cambios importantes y es imposible saber con certeza cuál va a ser el perfil definitivo de la industria. Sin embargo, dos desafíos se proyectan con claridad (DePaul, 2015). El mercado mundial de los minerales debe desarrollarse de manera que permita y no que limite la transición al desarrollo sostenible, básicamente mediante una internalización progresiva de los costos y, a la vez, manteniendo empresas viables y destacando las buenas prácticas. La creación de incentivos para la industria mediante soluciones de mercado debe ir a la par con la aplicación de normas y pautas. Los aspectos fundamentales del desarrollo sostenible deben ser incorporados a la cultura de las empresas mineras.

\section{RESULTADOS}

Algunos de los hallazgos en esta investigación surgieron desde la percepción de las personas entrevistadas: evidencia de deforestación de bosques de pino y hoja ancha, en el área del proyecto, polución provocada por la remoción de grandes cantidades de tierra, roca y acarreo de materiales, destrucción de lugares turísticos, contaminación de corrientes de agua por retención y descargas residuales, disminución severa en los niveles de agua superficiales y subterráneas, destrucción provocada por la extracción de arena de los lechos y cauces del río, erosión incontrolable en 
el área del proyecto minero, contaminación de pastos de los potreros aledaños a la mina. Los resultados, bajo la luz de las técnicas de recolección de datos, se definen de la siguiente manera.

Con relación a la esfera social, hay poco o ausencia de percepción de los beneficios sociales; como lo demuestra el grafico 1, la comunidad menciona que la minería provoca consumo de alcohol, tabaco y prostitución; la inversión en proyectos sociales se visualiza en materiales educativos y médicos; seis de cada diez personas estiman inversión en infraestructura; los pobladores opinan que la actividad minera tiene mucha incidencia en la vida social, relaciones familiares, nuevas costumbres y cambios de comportamiento con mayor presencia en hombres. También se observa que la emigración de la comunidad es moderada o poca; las consultas previas sobre concesiones a las comunidades de proyectos mineros son poco frecuentes; los poblados con mayor apoyo a la actividad minera son San Andrés y San Miguel; con tendencia negativa Azacualpa. Las enfermedades más frecuentes son dermatológicas, bronquiales e intestinales, las que son más comunes en San Andrés y Azacualpa; los pagos por servicios médicos en su mayoría son por cuenta de los pobladores; no se identifica la percepción de la indemnización por enfermedades por parte de la empresa minera.

\section{Grafico 1. Incidencia de problemas por operación minera}

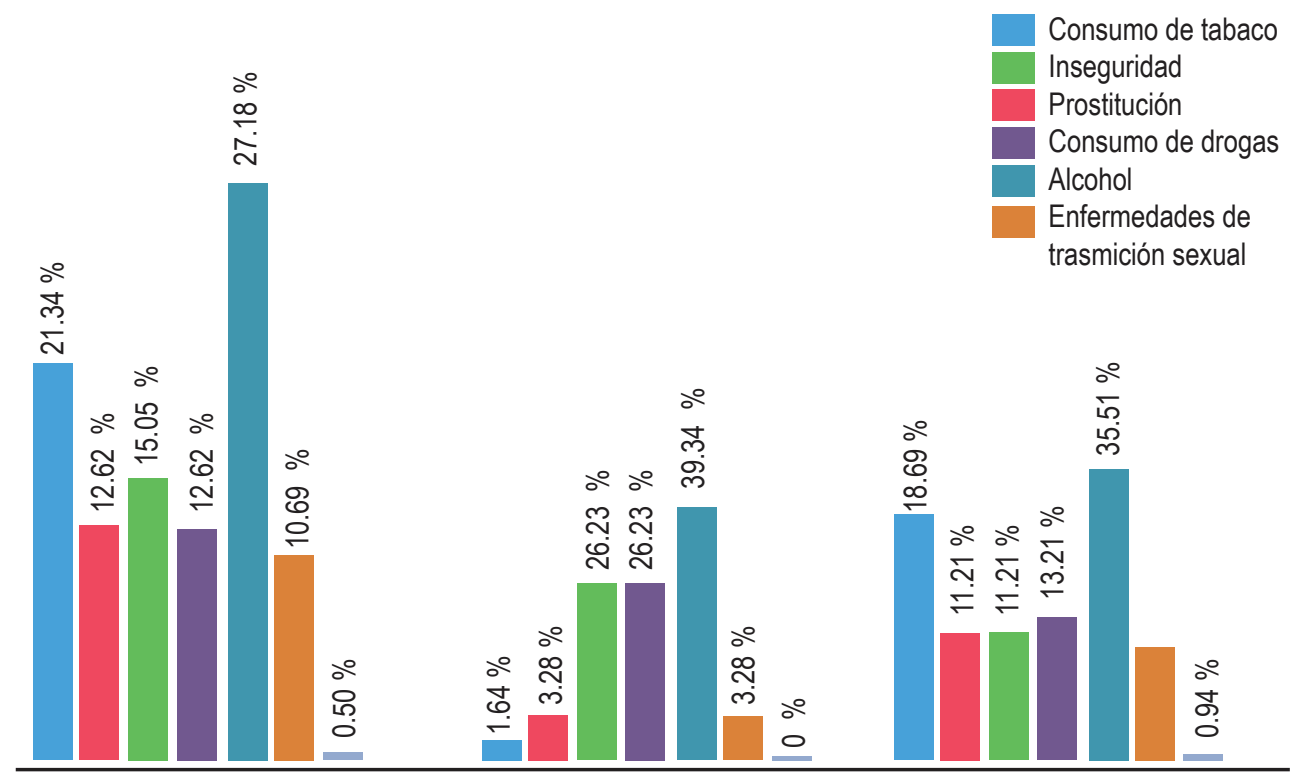

Azacualpa

San Miguel

San Andres

Fuente: Elaboración propia 
Con respecto a la dimensión económica, los pobladores encuestados de las comunidades de San Andrés y San Miguel opinan que la minería si ha contribuido a la economía del municipio en un $86.27 \%$ y $68.75 \%$ respectivamente. En contraste Azacualpa, opina que dicha empresa solo ha contribuido en un $43.02 \%$, según se puede observar en el grafico 2. Otras percepciones son que la empresa minera genera algunos empleos para sus pobladores, los contratos de trabajos se perciben frecuentemente equilibrados en oportunidades en las tres comunidades; aunque la frase con mayor identificación de las comunidades hacia la empresa minera es que ésta "hizo más daño que bien". La comunidad más beneficiada que se percibe en el estudio es San Andrés; se estima que los efectos de la minería perjudican a las comunidades, tomando en cuenta que esto es común en las todas las opiniones consultadas.

\section{Grafico 2. Ha contribuido la minería en la economía del municipio}

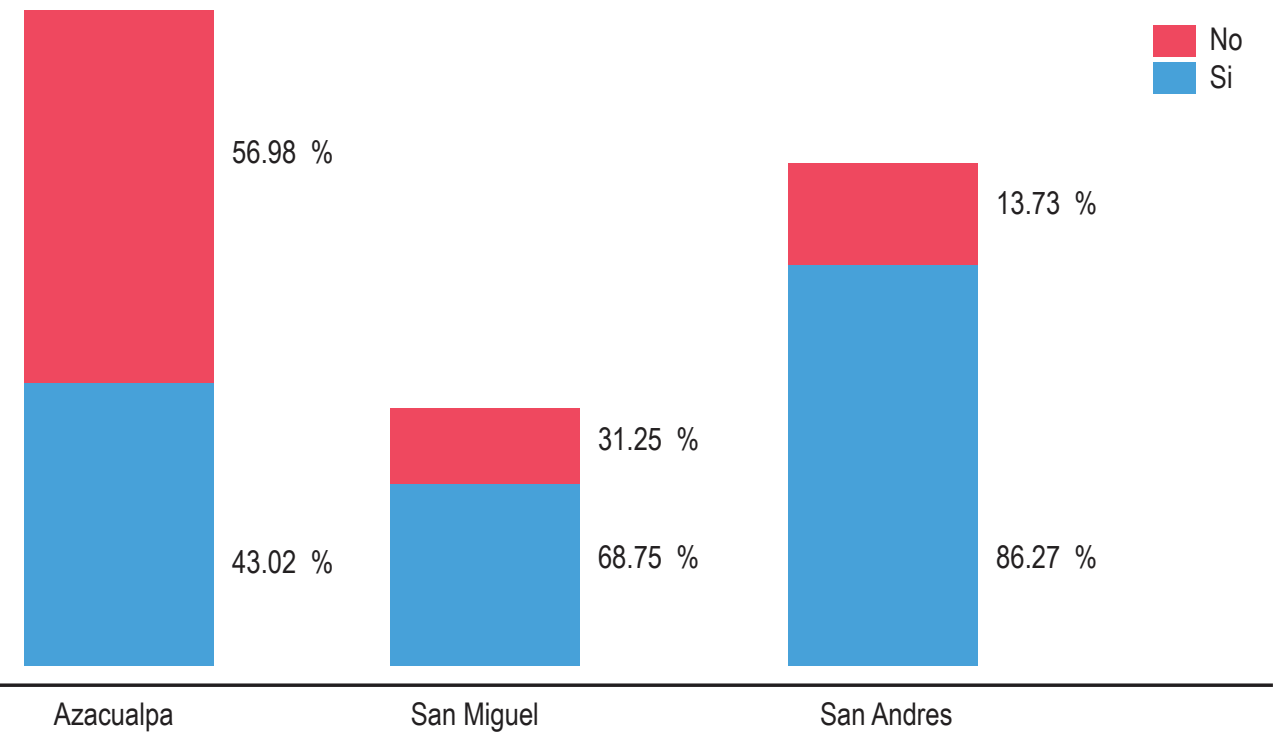

Fuente: Elaboración propia

Sobre la esfera ambiental, se estima que los efectos de la minería son muy desfavorables con alta incidencia en la contaminación del agua, deforestación de la flora, extinción de la fauna y destrucción del paisaje natural, como se puede observar en el grafico 3. Otros daños ambientales son polución provocada por la remoción de grandes cantidades de tierra y roca y acarreo de materiales, destrucción de lugares turísticos, contaminación de corrientes de agua por retención y descargas residuales 
en el rio Lara, afluente del Rio Higuito, disminución severa en los niveles de agua tanto superficiales como subterráneos, erosión incontrolable en el área del proyecto minero, contaminación de pastos de los potreros aledaños a la mina especialmente en la comunidad de San Miguel, entre otros.

\section{Grafico 3. Efectos ambientales provocados por la actividad minera}

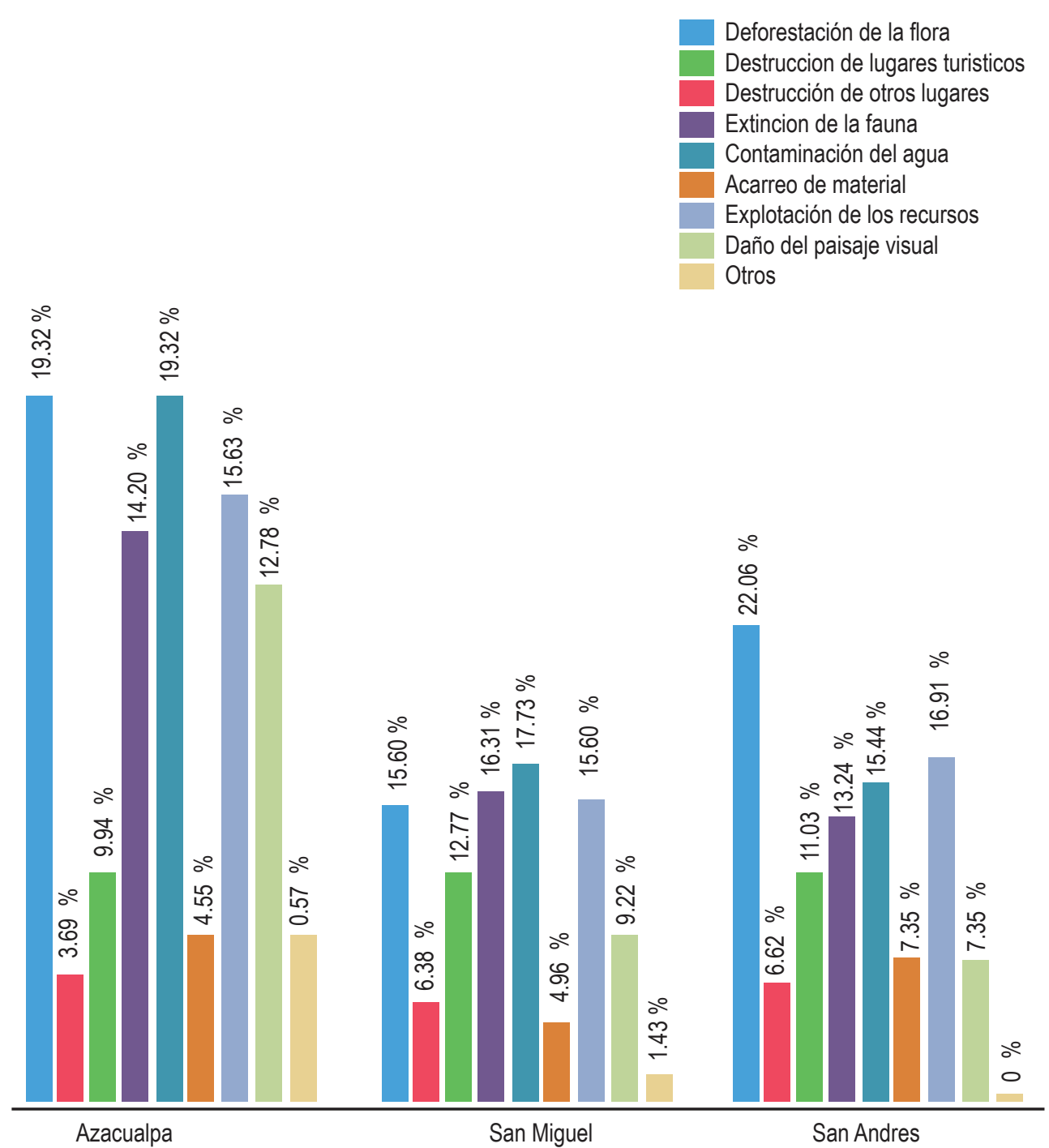

Fuente: Elaboración propia 


\section{DISCUSIÓN}

La industria de la minería enfrenta hoy día algunos de los desafíos más complejos que puede tener cualquier sector industrial, ya que es visto con desconfianza por muchos actores con las cuales trata a diario. Sin embargo, esta industria genera ciertas expectativas. Los países esperan que el desarrollo de la minería impulse un crecimiento económico sostenido, las comunidades locales esperan que la industria genere empleos, infraestructura y otros beneficios que contrarresten los riesgos e impactos que experimentan y, en definitiva, quedar en una mejor situación que al inicio del proyecto. Los empleados de la industria esperan contar con mejores condiciones de salud, seguridad laboral, con una mejor vida comunitaria y ser considerados al término de su fuente laboral; los ciudadanos locales y los defensores de los derechos humanos esperan que las empresas respeten y aporten los derechos básicos; las organizaciones ambientales esperan un mejor desempeño y que la industria evite operar en zonas delicadas en términos ecológicos y culturales.

Los inversionistas esperan mayores ingresos y han demostrado una notoria preocupación ante los resultados financieros de la industria; los consumidores esperan contar con productos seguros elaborados en procesos que cumplan con normas ambientales y sociales aceptables; también existen costos financieros asociados a la transición hacia el desarrollo sostenible. En algunos casos, estos costos pueden superar los beneficios de las mejoras. (Sandbrook, R. 2002). Aunque muchos sectores hablan de minimizar los impactos, en términos económicos el objetivo consiste en reducir los impactos hasta el punto en que los costos adicionales por la reducción de los impactos superen los beneficios adicionales. Por lo demás, los costos del desarrollo sostenible deben ser desglosados de tal modo que aseguren que las economías continúan siendo lo suficientemente viables para satisfacer las necesidades humanas de desarrollo y de diversos productos y servicios. Lo anterior, a su vez, implica que los precios que se pagan por los productos deben reflejar los verdaderos costos que supone suministrarlos.

Algunos cambios se lograrán mediante ganancias de eficiencia para todos, como la disminución en el uso de energía, pero la gran mayoría implicará internalizar costos que hasta ahora habían permanecido fuera del mercado. Por último, pero no por ello menos importante, el desarrollo sostenible también requiere de procesos democráticos que aseguren una participación de las personas en las decisiones que afectan sus vidas, así como de estructuras legales y políticas que garanticen el respeto de sus derechos civiles y políticos (Souza, 2005). Una gobernanza transparente y demo- 
crática confiere legitimidad al desarrollo y estimula a las organizaciones y empresas a rendir cuenta de sus acciones.

\section{CONCLUSIONES}

Los aportes económicos de la industria de la minería no son suficientes para resarcir los daños causados por la actividad minera en el medioambiente. Se estima que son más los costos sociales y ambientales que genera la industria de la minería que el beneficio económico. Los resultados demuestran también que la industria de la minería metálica no es compatible con el concepto universal del desarrollo que sugiere satisfacer las necesidades del presente, sin afectar las necesidades que necesitarán satisfacer las futuras generaciones; por cuanto la minería es un recurso no renovable. Ante esta situación, se plantean conclusiones orientadas al desarrollo humano sostenible en las comunidades de La Unión, Copán:

1. Dimensión económica: maximizar el bienestar humano, garantizar un uso eficiente de todos los recursos naturales a través de una optimización de las rentas, procurar identificar e internalizar los costos ambientales y sociales, mantener y mejorar las condiciones para la existencia de empresas viables.

2. Dimensión social: garantizar una distribución justa de los costos y beneficios del desarrollo entre todos los habitantes, respetar y reforzar los derechos fundamentales de los seres humanos, entre los que se incluyen las libertades civiles y políticas, la autonomía cultural, las libertades sociales y económicas y la seguridad personal, aspirar a mantener los avances en el tiempo. También garantizar que el agotamiento de recursos naturales no renovables no afectará a las futuras generaciones, mediante la sustitución de estos recursos por otras formas de capital.

3. Dimensión ambiental: fomentar una administración responsable de los recursos naturales y el medioambiente, incluyendo la reparación de los daños del pasado, reducir al mínimo los desechos y los daños ambientales en toda la cadena de abastecimiento, actuar con prudencia cuando los impactos sean desconocidos 0 inciertos, operar dentro de los límites ecológicos y proteger el capital natural fundamental.

4. Dimensión institucional: Fomentar la participación ciudadana de los pobladores de las comunidades mineras, especialmente en la consulta ciudadana libre, previa e informada antes del otorgamiento de concesiones mineras por parte de las instituciones del Estado de Honduras, y la participación de estas comunidades en todas las fases de exploración, explotación y de cierre de las empresas mineras. 


\section{AGRADECIMIENTOS}

A la Dirección de Investigación Científica y Posgrado de la Universidad Nacional Autónoma de Honduras, por permitirnos fortalecer nuestras capacidades en los temas de investigación; a las autoridades del Centro Regional Universitario de Occidente por toda su colaboración en el trabajo realizado; a la Coordinación Regional de Investigación Científica, a cargo del ingeniero Kevin Estévez y todo su equipo de trabajo. Asimismo, gracias a los estudiantes del CUROC, quienes contribuyeron con la recopilación de la información y a todas las personas que entrevistamos para este trabajo: líderes y consejeros de las comunidades de La Unión Copán.

\section{BIBLIOGRAFÍA}

Armiño, K. (2000).Diccionario de Acción Humanitaria y Cooperación al Desarrollo. España: Editorial Icaria.

Aráoz, A. (2009). El auge de la minería transnacional en América Latina. De la ecología política del neoliberalismo a la anatomía política del colonialismo. Quito: Ecuatoriana.

Bebbington, A. (2012). Extractive Industries, socio-environmental conflicts and political economic transformations in Andean America. London. Ed. Manchester.

DePaul, U. (2015). Conference Fragile World: Ecology \& the Church. Chicago: DePaul.

Gudynas, E. (2009). Diez tesis urgentes sobre el nuevo extractivismo: Contextos y demandas bajo el progresismo sudamericano actual. Uruguay: CLAES.

Gudynas, E. (2011). Buen vivir: germinando alternativas al desarrollo. Uruguay: ALAI. Leary, M. (2015). Pobreza: Panorama General, Banco Mundial. Recuperado de http://www.bancomundial.org/es/topic/poverty/overview

O’Neill, S. (2009). Percepciones de la Pobreza y el Bienestar Subjetivo en el Sur de Honduras. Tegucigalpa: UNAH-PLATS.

Power, T. (2009). Minería de metales y desarrollo sostenible en Centroamérica: Una valoración de costos y beneficios. Oxford: Oxfam América.

Pino, H. (2013). Diagnóstico de la situación minera en Honduras 2007-2012. Teguciglapa: ICEFI.

PNUD. (2011). Informe sobre Desarrollo Humano Honduras 2011. Reducir la inequidad: un desafío impostergable. Costa Rica: Litografía e Imprenta LIL. 
PNUD, (2013). Informe sobre Desarrollo Humano. Recuperado de http://www.hn.undp.org/content/honduras/es/home/presscenter/articles/2013/03/14/informesobre-desarrollo-humano-2013.html

Sandbrook, R. (2002). Minería, minerales y desarrollo sostenible. Recuperado de http://pubs.iied.org/pdfs/9287IIED.pdf

Salinas, H. (1993). ¿Hacia donde va la economía - mundo? Paris. Ed. BNF.

Santacruz, J. (2013). Oro y Finanzas. Recuperado de https://www.oroyfinanzas.com/2013/12/carta-editor-oro-cierra-2013-annus-horribilis/

Serrano, A. (2009). Migrando sobre la esfera: Pensar el desarrollo en la globalización desde América Latina. Tegucigalpa: Editorial Universitaria.

Souza, J. (2005). El arte de cambiar las personas que cambian las cosas: El cambio conceptual del ser humano desde su contexto cambiante. Brazilia: Red Nuevo Paradigma

\section{ANEXOS}

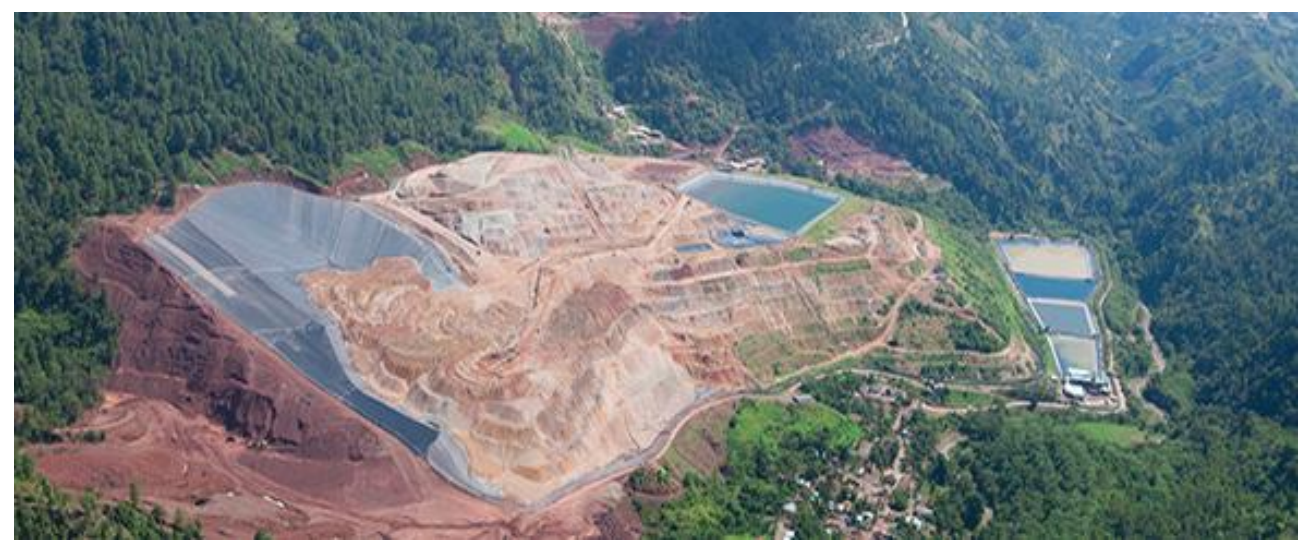

Foto: Patios de lixiviación de la mina San Andrés, La Unión Copán, Honduras 2015. 